\title{
The efficacy of sucrose for the relief of pain in neonates: a systematic review of the literature
}

\author{
Cláudia M. Gaspardo, ${ }^{1}$ Maria Beatriz M. Linhares, ${ }^{2}$ Francisco E. Martinez ${ }^{3}$
}

\begin{abstract}
Objective: To present a review of empirical studies published from 1993 to 2004 on the efficacy of sucrose solution for relieving pain in neonates.

Sources of data: Information was obtained from the MEDLINE/PsycINFO/ISI WEB of SCIENCE/LILACS and SCiELO databases by searching for "sucrose", "pain", "newborn" and "neonate".

Summary of data: Sucrose solution has demonstrated efficacy in pain relief during puncture procedures on samples of preterm and fullterm neonates. The recommendation is to administer oral sucrose, to the front of the tongue, 2 minutes before the painful procedure. Other non-pharmacological interventions, such as human breastmilk via nasogastric tube, non-nutritional suckling and being held at the breast, also demonstrated synergic analgesic effects when administered in association with sucrose. The majority of studies demonstrated a positive pain relief effect with a single $2 \mathrm{ml}$ dose at $25 \%$. Conclusions could not be drawn on the best repeat administration scheme; in the few studies that did provide an indication, the dose was $0.1 \mathrm{ml}$ at $24 \%$. The analgesic pain relief effect promoted by the sucrose was observed through altered behavioral responses, facial activity and crying. Heart rate underwent larger reductions as a result of sucrose administration during painful procedures than any of the other physiological responses.
\end{abstract}

Conclusions: The efficacy of a single dose of sucrose for the relief of acute pain neonates is well documented in the literature. However, there are not yet definite conclusions on the scheme of use for repeated doses of sucrose.

J Pediatr (Rio J). 2005;81(6):435-42: Pain, sucrose, neonates.

\section{Introduction}

The healthcare teams at neonatal intensive care units (Neonatal-ICU) often interpret pain response behavior as agitation, irritability or impatience. Neonates exhibiting such behavior are therefore treated with sedation, which is ineffective for reducing the perception of pain. ${ }^{1}$

1. MSc student, Faculdade de Medicina de Ribeirão Preto, Universidade de São Paulo (USP), São Paulo, SP, Brazil.

2. PhD. Professor, Department of Neurology, Psychiatry and Medical Psychology, Faculdade de Medicina de Ribeirão Preto, USP, São Paulo, SP, Brazil

3. Full professor and chief of the Puericulture and Pediatrics Department, Faculdade de Medicina de Ribeirão Preto, USP, SP, Brazil.

Manuscript received Apr 28 2005, accepted for publication Jun 292005.

Suggested citation: Gaspardo CM, Linhares MB, Martinez FE. The efficacy of sucrose for the relief of pain in neonates: a systematic review of the literature. J Pediatr (Rio J). 2005;81:435-42.
There is evidence that neonates have the neurological capacity to feel pain, even preterm neonates. The neurobiological system necessary for the perception of pain is formed between the 24th and 28th week of pregnancy, i.e., in preterms the peripheral and central structures necessary to sense pain are present and functioning at birth. Newborn infants may feel pain more intensely that older children and adults because their inhibitory control mechanisms are immature, which limits their capacity to modulate the painful experience. ${ }^{2}$

According to Grunau, ${ }^{2}$ exposure to pain is one of the most prejudicial factors in the extrauterine environment and can impact on cerebral development in many ways. For example, stress levels were evaluated by cortisol in the saliva of babies aged 8 months corrected, measured at baseline and after introducing a new toy. Among preterm babies who had been born with extremely low gestational ages, the levels of cortisol were significantly 
elevated before and after introduction of the toy, compared with children who had been born at older gestational ages and to full term. This hyper reactive behavior was associated with the large number of painful procedures to which the little preterms had been subjected, even after they had passed 8 months. ${ }^{3}$ Accepting that painful procedures are very common in the intensive care of preterm neonates, adequate assessment of reactions to pain and interventions to minimize them are tasks that are both relevant and necessary to the implementation of strategies to promote the development of newborns in Neonatal-ICUs.

There has been a considerable advance in studies aiming to establish indicators of neonatal pain, to validate pain and to measure the responses of preterm and fullterm neonates to pain ${ }^{8}$ which is a direct result of knowledge and understanding of the long term effects of early and repeated exposure of vulnerable neonates to painful experiences, ${ }^{2-5}$ effects such as lowering the pain threshold 2,6 and hyperalgesia. 2,7

Measurements that have been employed as indicators of pain in neonates with 28 or more weeks' gestational age include changes in facial activity, associated with the behavioral states of waking or sleep and physiological indices such as heart rate and oxygen saturation. ${ }^{9}$ It is recommended that the assessment of pain in newborn infants be multidimensional, utilizing physiological and behavioral measurements and thereby involving several different parameters. ${ }^{8}$

Pain relief increases the homeostasis and stability of newborn infants and is essential for the care and support of immature neonates so that they survive the stresses of Neonatal-ICUs. ${ }^{10}$ Intense pain should be managed with pharmacological agents, ${ }^{11}$ whereas lesser pain can be managed by means of non -pharmacological relief and prevention methods. ${ }^{10-12}$

A selection of non-pharmacological interventions are employed for pain relief in neonates, such as, for example, giving pacifiers, changing position, cradling in the arms, swaddling, maintaining the body in a curved position, giving postural support and reducing tactile stimulation, have all been used for pain management during procedures in order to facilitate the organization and autoregulation of preterm neonates. ${ }^{12,13}$ Nevertheless, there are still many points of disagreement and controversies surrounding the most effective form of pain relief. ${ }^{14}$ For example, authors have demonstrated that significant alterations take place to both behavioral and physiological responses during capillary puncture for blood testing irrespective of the position of the neonate in the incubator, whether decubitus ventral or dorsal. ${ }^{15}$

The use of sweetened solutions for the management of painful procedures, in particular sucrose, has also been recommended and widely studied.6,16 Notwithstanding, non-pharmacological acute pain relief during invasive and painful procedures at Neonatal-ICUs still needs to be better assessed.
The objective of this study was to perform a systematic review of indexed literature published from 1993 to 2004 and relating to experimental studies assessing evidence on the effect of sucrose for acute pain relief in neonates during puncture procedures for blood sampling.

\section{Methods}

A systematic search was performed of the literature by means of consulting the research indexers of electronic databases (MEDLINE, PsycINFO, ISI WEB of SCIENCE, LILACS and SciELO). The query employed the following keywords: sucrose/sacarose AND/E pain/dor AND/E newborn (OR neonate)/recém-nascido (OU neonato). Inclusion criteria were defined as: experimental articles, in English, Portuguese or Spanish, undertaken with human beings in the neonatal phase, published from January 1993 to May 2004 in specialist periodicals and indexed by the databases consulted.

Seventy-nine articles were returned and of these 50 were excluded from the analysis. Twenty-five articles were excluded because they assessed other interventions for pain relief in neonates, seven articles because they dealt with older infants and children, six that involved research with animals, five that were on circumcision, four that were review articles, two that didn't assess responses to pain and one article was excluded that dealt with crying as a measure of pain in neonates, but without sucrose administration. The remaining 29 articles were located and included in this review.

Table 1 illustrates the process of selection for the articles to be reviewed.

\section{Results}

Sixteen of the 29 articles selected were studies of fullterm neonate patient samples, ${ }^{17-32}$ eleven studied preterm neonates ${ }^{33-43}$ and two included comparisons between preterm and fullterm neonates. 44,45

The volume of sucrose used in the majority of the studies involving fullterm neonates was $2 \mathrm{ml}, 17-25,27-32$ with concentrations of $12 \%, 20,24,30,3112.5 \%, 18,3224 \%, 27$ $25 \%, 18,19,21,2930 \% 23,25$ and $50 \% .18,19,22$ Four studies $17-19,28$ compared different concentrations of sucrose.

The volume used in studies performed with preterm neonates varied more, with studies of single dose interventions administering $0.05 \mathrm{ml}^{36} 1 \mathrm{ml}^{35,40}$ and $2 \mathrm{ml}^{33,34,42}$ and studies of repeated doses used $0.05 \mathrm{ml}^{39}$ and $0.1 \mathrm{ml} .37,40,43$ The concentrations administered were $15 \%, 4024 \%, 3725 \% 38,42$ and $50 \% 33$ in single-dose studies, and $24 \% 39,41,43$ in studies of repeat doses. Two studies 34,40 assessed the sucrose solution at varying volumes and concentrations. The two studies with samples including both preterm and full term neonates $44,450.5 \mathrm{ml}$ of sucrose was administered at a concentration of $24 \%$.

In 26 studies $^{17-38,40-42,44}$ (90\%) the sucrose solution was administered in a single dose only before painful 
Table 1 - Process of articles selection

\begin{tabular}{lc}
\hline Process of articles selection & Number \\
\hline Total of articles identified & 79 \\
Total of articles excluded & 50 \\
$\quad$ Review articles & 46 \\
$\quad$ Articles that did not comply with the study goal & 46 \\
Total of articles selected & 29 \\
Total of articles found & 29 \\
\hline
\end{tabular}

procedures. Just three studies, $39,41,43$ performed on preterm neonates, administrated sucrose solution in repeat doses and more than once during the same painful procedure ${ }^{39}$ or during all painful procedures undergone by the neonate over a seven-day period, during their stay at the NeonatalICU. ${ }^{41,43}$ In just one study ${ }^{43}$ the solution was administered to a location other than the anterior region of the neonate's tongue, in order to avoid possible aspiration.

The instrument used to assess responses to pain in four $37,39,42,44$ studies was multidimensional and had been validated for the evaluation of neonatal pain, the Premature Infant Pain Profile (PIPP). The instrument assesses facial expression, heart rate, oxygen saturation, behavior and gestational age and returns a single overall score.

Eleven studies 18,19,21,24,26,30,32,35,36,41,42 assessed facial actions (brow bulge, eye squeeze, nasolabial furrow, open lips, mouth stretch (horizontal and vertical), lip purse, tongue tautening, and chin quiver, ) using the Neonatal Facial Coding System (NFCS), which is a one-dimensional instrument validated for evaluation of the response to neonatal pain. Two studies ${ }^{19,39}$ evaluated body movements (upper and lower limbs) using a validated one-dimensional instrument, the Infant Body Coding System (IBCS). Carbajal et al. ${ }^{23}$ used a validated, multidimensional behavioral instrument for behavioral analysis of neonates called the Douleur Aiguë du Nouveau-né (DAN) and which evaluates facial expression, vocal expression and body movements.

Other supplementary physiological and behavioral indicators were also used to assess reactions to pain, for example vagal tone, 24,31 saliva cortisol, 31,43 skin conductance, 40 respiratory frequency, $29,33,34$ crying 18-21,24-27,29-32,34,35,38,40,42 and sleep/wake state. $21,23,40$

\section{Modes of sucrose administration}

Single dose administration

Sucrose and sterile water control

Eight studies 17,18,29,33-35,38,42 compared the administration of a sucrose solution 2 minutes before a painful puncture procedure with administration of sterile water (control). Administering sucrose solution at $25 \% 29,35,38,42$ and $50 \% 33$ reduced the neonates' heart rate, 29,33 time crying $29,33,38$ and level of facial activity as measured by the Neonatal Facial Coding System (NFCS) $29,35,38$ in response to painful procedures when compared with sterile water. In a study carried out by Ramenghi et al., 38 the sucrose solution was administered before capillary puncture to a sample of preterm neonates, on one occasion via oral route and on another via nasogastric tube, with the order of administration route being randomized in order to observe pre and post absorption effects.

\section{Concentration of sucrose solutions}

With relation to the issue of the most effective concentration of sucrose for pain relief in neonates, Blass $\&$ Shah $^{17}$ found that a sucrose solution was more effective at $0.17 \mathrm{M}$ than at $0.34 \mathrm{M}$ at reducing the crying time of fullterm neonates, during and after taking blood. Furthermore, the same study also concluded that the most effective interval between sucrose administration and starting the painful procedure was 2 minutes. Haouri et al. ${ }^{18}$ found that sucrose at $50 \%$ was more effective at reducing time crying and heart rate after a painful procedure than sucrose at 12.5 and $25 \%$ and sterile water with fullterm neonates. Abad et al. ${ }^{34}$ found that sucrose at $12 \%$ exhibited a greater reduction in the heart rates of preterm neonates than either sucrose at $24 \%$ or sterile water. In contrast, the $24 \%$ sucrose solution provoked a greater reduction in crying time than either sucrose at $12 \%$ or sterile water, after the painful procedure.

\section{Sucrose solution versus glucose}

Sucrose solution has also been compared with glucose administration for efficacy of acute relief of the pain from venous puncture in fullterm neonates. ${ }^{25,28}$ Results reported by Isik et al. ${ }^{25}$ demonstrated that fullterm neonates given $2 \mathrm{ml}$ of sucrose solution at $30 \%$ exhibited a significant reduction in time crying during the painful procedure when compared with groups that received glucose at 10 and $30 \%$ 
and a control group. While there was no significant difference between the groups in terms of heart rate, the difference between baseline and post-puncture was smaller in the sucrose solution group.

A study by Guala et al. ${ }^{28}$ also failed to observe significant differences in change in heart rate from baseline during a painful procedure between groups of fullterm neonates who were given $2 \mathrm{ml}$ of glucose at 5, 33 and $50 \%$ and groups who were given sucrose at 33 and $50 \%$ and sterile water. Nevertheless, the groups given glucose at 33 and $50 \%$ exhibited lesser increases from baseline heart rate after a procedure when compared with sucrose and control groups, contradicting the results of the previous study.

\section{Sucrose solution and human milk}

Sucrose solution administered 2 minutes before a painful puncture procedure has also been compared with administering human milk. Örs et al. ${ }^{21}$ found that fullterm neonates who were given $2 \mathrm{ml}$ of sucrose solution at $25 \%$ exhibited a significant reduction in time crying during a capillary puncture procedure compared with neonates who were given human milk and a control group given water. Furthermore, the authors found that the group that was given sucrose solution exhibited a shorter mean recovery time and a lower increase on baseline heart rate during the puncture, in comparison with the other two groups.

Storm \& Fremming ${ }^{40}$ carried out a study that, in addition to assessing the effect of human milk on the response to pain in preterm neonates, also compared the effect of the milk administered in association with sucrose solution and also of sucrose solution at two different dosages and concentrations. Their results indicated that the time spent crying was reduced in the groups of neonates given $1 \mathrm{ml}$ of sucrose at $25 \%$ by mouth and human milk via nasogastric tube, compared with groups given $2 \mathrm{ml}$ of sucrose solution at $15 \%$ and a control group; thus demonstrating less efficacy with a concentration of $15 \%$ than with $25 \%$. It should be pointed that those neonates who were given human milk via nasogastric tube followed by $1 \mathrm{ml}$ of sucrose at $25 \%$ by mouth cried for shorter periods and were less agitated than the other groups.

\section{Sucrose solution, human milk and breastfeeding}

One study 29 investigated the efficacy of sucrose and breastfeeding for reducing the pain of fullterm neonates undergoing capillary puncture and found that crying time was significantly reduced among neonates given $2 \mathrm{ml}$ of sucrose solution at $25 \% 2$ minutes before the painful procedure, compared with groups of neonates who were breastfed or given sterile water (control). The group given sucrose also exhibited a significant reduction in recovery time compared with groups given human milk via syringe or sterile water, and a significant reduction in IBCS scores compared with the groups given human milk via syringe, breastfeeding or sterile water. The same study also assessed possible adverse events caused by administering sucrose solution, by means of assessing the development of necrotizing enterocolitis and did not detect any such link.

\section{Sucrose solution versus non-nutritive sucking}

Blass \& Watt ${ }^{24}$ performed a comparative study of four groups, the members of which were treated as follows: sucrose solution at $12 \%$, administered 2 minutes before the puncture procedure, sterile water, sucrose associated with non-nutritive sucking (pacifier) and sterile water associated with non-nutritive sucking. Both sucrose and non-nutritive sucking, demonstrated independent analgesic effects when each was compared with water. It was sucrose in association with non-nutritive sucking, however, that demonstrated the greatest analgesic effect of all four groups. It should be pointed out that non-nutritive sucking with sterile water exhibited an analgesic effect, identified during the painful procedure by the absence of crying and by facial expression, only for those fullterm neonates whose rate of suction was more than 32 /minute.

Two studies 44,45 looked at the same sample composed of preterm and fullterm neonates in order to assess the efficacy of sucrose solution $(0.5 \mathrm{ml}$ at $24 \%)$ compared with non-nutritive sucking for relieving capillary puncture procedural pain 44 and to verify the influence of gestational age on the efficacy of such interventions. ${ }^{45}$ Gibbins et al. ${ }^{44}$ observed that groups of preterm and fullterm neonates given a sucrose solution followed by non-nutritive sucking 2 minutes before a puncture procedure exhibited a significant reduction in PIPP scores after a painful procedure when compared with groups of neonates given just sucrose solution or sterile water followed by non-nutritive sucking.

Gibbins \& Stevens 45 found that a sucrose solution followed by non-nutritive sucking was the most effective intervention at reducing PIPP scores during punctures for three groups of neonates with gestational ages of 27 to 31 weeks, 32 to 35 weeks and 36 to 42 weeks, when compared with sucrose alone or sterile water followed by non-nutritive. In addition to this, their results revealed that the physiological and behavioral responses to painful stimuli are of greater magnitude in more mature neonates and that in less mature neonates the administration of sucrose caused a greater number of adverse effects (coughing and vomiting). These effects did not attain statistical or clinical significance, which supports the short-term safety of administering sucrose.

Sucrose solution compared with other non-pharmacological (glucose, colostrum, dextrose or granulated sugar), associated or not with non-nutritive sucking

Carbajal et al. ${ }^{23}$ reported that non-nutritive sucking had greater efficacy for the relief of pain in fullterm neonates during venous puncture than either glucose or sucrose. Furthermore, the analgesic effects of $2 \mathrm{ml}$ of both glucose and sucrose at $30 \%$ were equally significant in comparison with the group given sterile water and the untreated group, according to the DAN behavioral assessment instrument. There was a tendency towards lower DAN scores in the non- 
nutritive sucking plus sucrose solution group than the nonnutritive sucking in isolation group.

Blass \& Miller ${ }^{30}$ demonstrated that colostrum, given by syringe or pacifier did not reduce the duration of crying or facial activity of fullterm neonates at the point of capillary puncture, when compared with a control group given sterile water. However, colostrum administered with a pacifier did prevent heart rate from increasing at the point of painful stimulus. In contrast, $2 \mathrm{ml}$ of sucrose at $12 \%$ administered by syringe or pacifier reduced the duration of crying, reduced facial activity and attenuated the increase in heart rate at the point of puncture, compared with colostrum and sterile water.

Akman et al. ${ }^{32}$ also found that fullterm neonates given $2 \mathrm{ml}$ of sucrose solution at $12.5 \%$ followed by nonnutritive sucking cried less and presented lower facial activity scores by NFCS during a capillary puncture procedure when compared with neonates given sucrose, dextrose, dextrose followed by non-nutritive sucking and sterile water (control).

Greenberg ${ }^{31}$ performed a study with the objective of examining the efficacy of non-nutritive sucking and sugars for the management of capillary puncture procedural pain in fullterm neonates. A group given granulated sugar on a pacifier exhibited shorter duration of crying compared with a group given sterile water and pacifier with water. Vagal tone was also reduced during the painful procedure in the group given pacifier with granulated sugar compared to groups given sterile water and $2 \mathrm{ml}$ of oral sucrose solution at $12 \%$.

Sucrose administrated with non-nutritive sucking compared to baby positioning

Stevens et al. ${ }^{37}$ found a significantly reduced PIPP score during painful procedures in preterm neonates given a pacifier with sucrose and a pacifier with sterile water, compared with neonates kept in the prone position or receiving no intervention. They also observed a tendency towards a lower PIPP score among neonates given a pacifier with sucrose compared with a group given a pacifier with water. In this study the prone position did not exhibit efficacy at reducing the response to pain as measured by the PIPP. In terms of contextual factors, the frequency of painful procedures showed a positive correlation with PIPP score, i.e. the more painful procedures a neonate suffered, the higher their PIPP score.

\section{Sucrose and contact at the breast}

Two studies included positioning fullterm neonates at the breast of an adult during the painful procedure of capillary puncture. Overgaard \& Knudsen 22 found that neonates given $2 \mathrm{ml}$ of sucrose solution at $50 \% 2$ minutes before puncture and were held cried significantly less during the painful procedure compared with a group given sterile water and held.

In a similar manner, Gormally et al. 26 identified a reduced duration of crying during a capillary puncture procedure among a group of fullterm neonates given 2.5 $\mathrm{ml}$ of sucrose at $24 \%$ and being and a group given just sucrose compared with a group given sterile water and held and another given just water. Heart rate was only reduced in the group of neonates given sucrose and held. This study reaffirmed the efficacy of sucrose for acute pain relief since the administration of sucrose alone was enough to reduce the duration of crying.

\section{Sucrose solution and anesthetic cream}

Just one study related sucrose solution with a pharmacological intervention for the study of pain, with the agent concerned being a eutectic mixture of prilocaine and lidocaine EMLA for topical use. In this study, Abad et al. 27 identified a significant reduction in heart rate and duration of crying in groups of fullterm neonates who, 2 minutes before a venous puncture procedure, received $2 \mathrm{ml}$ of sucrose at $24 \%$ in isolation or combined with $1 \mathrm{~g}$ of EMLA anesthetic cream after the painful procedure and compared with a group given sterile water. Furthermore, the EMLA cream when applied in isolation was less effective than either the sucrose solution administered alone or in combination with EMLA cream. The results also revealed that using topical EMLA anesthetic cream did not increase the analgesic effect of the sucrose solution.

\section{Administration of sucrose solution in repeated doses}

Three studies $39,41,43$ performed by a single team of Canadian researchers employed sucrose solution in repeated doses and all of these were performed with preterm neonates who were compared with a control group given sterile water.

In the study by Johnston et al., ${ }^{39}$ repeated doses were administered three times during a single painful capillary puncture procedure, 2 minutes before the procedure, at the exact moment of the procedure and 2 minutes after the painful procedure. The neonates were randomized into three groups, who were given either, sucrose solution for the first dose and sterile water for the next two, three sucrose solution doses or three doses of sterile water. The results revealed that the groups of neonates who were given $0.05 \mathrm{ml}$ of sucrose solution at $24 \%$, both in single and triple doses had lower PIPP scores than the group given water. The study also showed that, during the last 30 seconds of evaluation of the response to the painful procedure, during recovery, neonates who had been given repeated doses of sucrose exhibited lower PIPP scores than those given a single dose.

In the study authored by Johnston et al. ${ }^{41}$ neonates were randomized into treatment groups and given sucrose solution, or a control group, given sterile water, before every invasive procedure for seven days. Their results revealed that $0.1 \mathrm{ml}$ of sucrose solution at $24 \%$, administered in repeated doses, exhibited efficacy for reducing NFCS scores during capillary puncture, venous puncture and aspiration. In terms of possible adverse effects of sucrose, the authors observed that a high number of sucrose doses was a predictor of a low neurobehavioral development 
score, for motor and vitality, alertness and orientation on the Neurobehavioral Assessment of the Preterm Infant (NAPI) scale, in babies with postconceptional ages of 36 and 40 weeks and high Neuro-Biological Risk Scores (NBRS) in neonates with two weeks' post-gestational age.

Boyer et al. ${ }^{43}$ set themselves the objective of evaluating the efficacy for physiological stability of administering sucrose solution for all painful procedures. The same sample and procedures as described immediately above were employed. This study found a significant negative correlation between the standard deviation for heart rate and the number of doses of sucrose, in those neonates who had received a large number of sucrose solution doses (nine doses or more within 24 hours), i.e. the higher the number of sucrose solution doses, the lower the standard deviation for heart rate.

\section{Critical analysis of the methodology employed}

It is of note that all studies included a control group in their study samples, generally given sterile water, which is a relevant methodological precaution when assessing the efficacy of sucrose solution for pain relief with neonates. With a single exception, all of the studies employed randomization for assigning patients into study groups. Two studies that compared sucrose with other nonpharmacological interventions exhibited a lack of control of non-nutritive sucking in their study design, making interpretation of their data problematic.

A fair proportion of the studies (55\%) employed validated instruments to assess the pain of fullterm and preterm neonates. In all of these instrument scores were produced by observers blinded to the study objective and the interventions employed. One further methodological precaution that merits attention is the assessment of pain indicators at baseline, during the painful procedure and recovery, undertaken in $83 \%$ of the studies.

The majority of the studies ( $72 \%)$ included multidimensional analyses combining the findings from one-dimensional procedures to assess the response to pain, each focussing on either physical or behavioral indicators, as was recommended by Craig et al. ${ }^{8}$ More studies assessed single dimensions for later combination during analysis rather than making assessments with a multidimensional instrument such as the PIPP. This last combines indicators of different types into a single score, with behavioral indicators such as facial activity and sleep/wake state, physiological indicators such as heart rate and oxygen saturation and neonatal health status such as gestational age, which can be make it difficult to interpret results. The advantage of using a variety of dimensions gauged according to different information sources is that there is the possibility of neutralizing the limitations of each particular measurement and may enrich the analysis of the standards of neonate reactivity by differentiating effects according to behavioral and physiological indicators.

Just three studies found that sucrose had lesser efficacy than other non-pharmacological interventions. ${ }^{23,28,31}$ The first, ${ }^{23}$ found that non-nutritive sucking was more effective than sucrose solution for pain relief with fullterm neonates. this study, however, assessed pain response using just behavioral parameters, which can be characterized as a limitation to the study due to the lack of physiological parameters.

In the second of these studies, ${ }^{28}$ glucose was observed to have greater efficacy than sucrose. It must be kept in mind, however, that this study had a limitation in terms of the techniques for assessment of pain which was based purely on the physiological indicator heart rate, with no behavioral indicators analyzed, which have been demonstrated as being more sensitive in terms of the analgesic effects of sucrose. Furthermore, this study employed sucrose at concentration of 33 and $50 \%$, whereas the concentration shown to be most effective by the majority of studies is $25 \%$.

The third of these studies ${ }^{31}$ reported greater pain relief efficacy for non-nutritive sucking together with granulated sugar than for sucrose solution with fullterm neonates. Since one of the physiological indicators evaluated by this study was saliva cortisol, it was necessary to administer an acid substance by mouth to produce saliva, which in turn may have modified the infants' perception of the sweet taste of sucrose. Another point that is worth noting with relation to this study is that, in order to avoid any possibility of aspiration, the sucrose solution was administered to one side, at the corner of the babies' mouths, whereas the recommendation is to administer the solution to the anterior region of the tongue, where the taste buds responsible for identifying sweetness are located.

Few studies (25\%) assessed the possible adverse effects caused by administering sucrose, such as coughing, vomiting, abdominal distension or necrotizing enterocolitis, although none of those that did returned significant results for these variables.

Just $21 \%$ of the studies concerned themselves with controlling for the number of invasive and painful procedures undergone by the neonates in their samples. One of the studies that did account for this variable found a significant relationship between it and the neonates' reactions to pain, indicating that the greater number of painful procedures undergone by a neonate, the higher their score on the PIPP scale. Control of this variable should be taken into account when selecting sample groups or during data analysis in order to guarantee more reliable results.

\section{Conclusions}

Sucrose solution demonstrates efficacy for the relief of procedural pain from capillary or venous puncture in healthy full and preterm neonates. There is consensus across the results that the solution should be administered orally, 2 minutes before the painful procedure, to the anterior portion of the neonate's tongue.

Some other non-pharmacological interventions, human milk via nasogastric tube, non-nutritive sucking and holding at the breast exhibited a synergic analgesic effect with sucrose solution when administered in combination. 
Both for fullterm and preterm neonates, a single $2 \mathrm{ml}$ dose of sucrose solution at $25 \%$ demonstrated a positive result in the majority of studies. There is not yet, however, a defined dosage for repeated administration, although the scheme most used in these studies was $0.1 \mathrm{ml}$ at $24 \%$.

The indicators used for response to pain that demonstrated greatest sensitivity for sucrose solution during painful procedures were behavioral, in particular facial activity and crying. The physiological indicator heart rate was also altered by the administration of sucrose solution, particularly in terms of reduced time to recovery. Changes were not, however, observed in oxygen saturation and saliva cortisol.

Despite the American Academy of Pediatrics and the Canadian Paediatric Society 16 recommending the use of sucrose solution as routine pain relief in Neonatal-ICUs, during invasive and painful procedures, studies into the use of this substance in repeat doses remain inconclusive and merit fresh investigation. In these, assessment of the efficacy of administering repeated doses of sucrose for the relief of pain in neonates must by rights widen the study of the possible side effects, both from the point of view of health and of development, in addition to investigating how to control the number of invasive procedures.

\section{Acknowledgements}

We are grateful for the contributions made by Vivian C. Klein, Juliana T. Chimello (Masters students in Mental Health at the Ribeirão Preto Medical Faculty of the Universidade of São Paulo) and by Thaís S. Cugler (Undergraduate Medical Student at the Ribeirão Preto Medical Faculty of the Universidade of São Paulo), reading and synthesizing a large number of studies and organizing and writing up the results.

\section{References}

1. Broome M, Tanzillo H. Differentiating between pain and agitation in premature neonates. Perinato Neonatal Nurs. 1990;4:53-2.

2. Grunau R. Early pain in preterm infants: a model of long-term effects. Clin Perinatol. 2002;29:373-94.

3. Grunau R, Weinberg J, Whitfield M. Neonatal procedural pain and preterm infant cortisol response to novelty at 8 months. Pediatrics. 2004;114:77-84.

4. Anand K. Effects of perinatal pain and stress. In: Mayer E, Saper C. Progress in Brain Research. Amsterdam: Elsevier Science; 2000. p. 117-29.

5. Grunau R. Long-term consequences of pain in human neonates. In: Anand K, Stevens B, McGrath P. Pain in Neonates. Netherlands: Elsevier Science; 2000. p. 55-76.

6. Gibbins S, Stevens B, Asztalos E. Assessment and management of acute pain in high-risk neonates. Pharmacother. 2003;4: 475-83.

7. Anand K. Clinical importance of pain and stress in preterm neonates. Biol Neonate. 1998;73:1-9.

8. Craig K, Whitfield M, Grunau R. Pain in the preterm neonate: behavioral and physiological indices. Pain. 1993;52:287-99.

9. Morrison S, Grunau R, Oberlander T, Whitfield M. Relationships between behavioral and cardiac autonomic reactivity to acute pain in preterm infants. Clin J Pain. 2001;17:350-8.

10. Corff K, Seidman R, Venkataraman M. Facilitated tucking: a nonpharmacological comfort measure for pain in preterm neonates. J Obstetric Gynecol Neonatal Nurs. 1995;24:143-5.
11. Stevens B, Gibbins S, Franck L. Treatment of pain in the neonatal intensive care unit. Pediatr Clin North Am. 2000;47: 633-50.

12. Franck L, Lawhon G. Environmental and behavioral strategies to prevent and manage neonatal pain. Semin Perinat. 1998;22: 434-43.

13. 13. Franck $L$, Miakowski C. Measurement of neonatal responses to painful stimuli: a research review. J Pain Symp Manag. 1997; 14:343-78.

14. Anand K, Dphil M. Consensus statement for the prevention and management of pain in the newborn. Arch Pediatr Adolesc Med. 2001;155:173-9.

15. Grunau R, Linhares MB, Holsti L, Oberlander T, Whitfield M. Does prone or supine position influence pain responses in preterm infants at 32 weeks gestational age? Clin J Pain. 2004;20:76-82.

16. American Academy of Pediatrics \& Canadian Pediatric Society. Prevention and management of pain and stress in the neonate. Pediatrics. 2000; 105:454-61.

17. Blass $E$, Shah A. Pain-reducing properties of sucrose in human newborns. Chem Senses. 1995;20:29-35.

18. Haouri N, Wood C, Griffiths G, Levene M. The analgesic effect of sucrose in full term infants: a randomized controlled trial. BMJ. 1995;3:1451-1500.

19. Ramenghi L, Griffith G, Wood C, Levene M. Effect of non-sucrose sweet tasting solution on neonatal heel prick responses. Arch Dis Child Fetal Neonatal Ed. 1996;74:129-31.

20. Blass E. Milk-induced hypoalgesia in human newborns. Pediatrics. 1997;99:825-9.

21. Örs R, Özek E, Baysoy G, Cebeci D, Bilgen $H$, Turkuner M, et al. Comparison of sucrose and human milk on pain response in newborns. Eur J Pediatr. 1999;158:63-6.

22. Overgaard C, Knudsen A. Pain-relieving effect of sucrose in newborns during heel prick. Biol Neonate. 1999;75:279-84.

23. Carbajal R, Chauvet $X$, Couderc S, Olivier-Martin M. Randomized trial of analgesic effects of sucrose, glucose, and pacifiers in term neonates. BMJ. 1999;319:1393-7.

24. Blass M, Watt B. Suckling and sucrose-induced analgesia in human newborns. Pain. 1999;83:611-23.

25. Isik U, Ozek E, Bilgen $H$, Cebeci D. Comparison of oral glucose and sucrose solutions on pain response in neonates. J Pain. 2000;1:275-8.

26. Gormally S, Barr R, Wertheim L, Alkawaf R, Calinoiu N, Young $S$. Contact and nutrient care giving effects on newborn infant pain responses. Dev Med Child Neurol. 2001;43:28-38.

27. Abad F, Diaz-Gomez N, Domemech E, Gonzalez D, Robayna M, Feria M. Oral sucrose compares favorably with lidocaine-prilocaine cream for pain relief during venopuncture in neonates. Acta Paediatr. 2001;90:160-5.

28. Guala A, Pastore G, Liverani M, Giroletti G, Gulino E, Meriggi A, et al. Glucose or sucrose as an analgesic for newborns: a randomized controlled blinded trial. Minerva Pediatr. 2001;53:271-4.

29. Bilgen $H$, Ozek $E$, Cebeci $D$, Ors R. Comparison of sucrose, expressed breast milk, and breast-feeding on the neonatal response to heel prick. J Pain. 2001;2:301-5.

30. Blass E, Miller L. Effects of colostrum in newborn humans: dissociation between analgesic and cardiac effects. J Dev Behav Pediatr. 2001;22:385-90.

31. Greenberg C. A sugar-coated pacifier reduces procedural pain in newborns. Pediatr Nurs. 2002;28:271-7.

32. Akman I, Ozek E, Bilgen H, Ozdogan T, Cebeci D. Sweet solutions and pacifiers for pain relief in newborn infants. J Pain. 2002;3: 199-202.

33. Bucher H, Moser T, Siebenthal K, Keel M, Wolf M, Duc G. Sucrose reduces pain reaction to heel lancing in preterm infants: $A$ placebo-controlled, randomized and masked study. Pediatr Res. $1995 ; 3: 332-5$.

34. Abad F, Díaz N, Domenech E, Robayna M, Rico, J. Oral sweet solution reduces pain-related behavior in preterm infants. Acta Paediatr. 1996;85:854-8.

35. Ramenghi L, Wood C, Griffith G, Levene M. Reduction of pain response in premature infants using intraoral sucrose. Arch Dis Child Fetal Neonatal Ed. 1996;74:126-8.

36. Johnston C, Stremler R, Stevens B, Horton L. Effectiveness of oral sucrose and simulated rocking on pain response in preterm neonates. Pain. 1997;72:193-9. 
37. Stevens B, Johnston C, Franck L, Petryshen P, Jack A, Foster G. The efficacy of developmentally sensitive interventions and sucrose for relieving procedural pain in very low birth weight neonates. Nurs Res. 1999;48:35-43.

38. Ramenghi L, Evans D, Levene M. "Sucrose analgesia": absorptive mechanism or taste perception? Arch Dis Child Fetal Neonatal Ed. $1999 ; 80: 146-7$.

39. Johnston C, Stremler R, Horton L, Friedman A. Effect of repeated doses of sucrose during heel stick procedure in preterm neonates. Biol Neonate. 1999;75:160-6.

40. Storm $H$, Fremming $A$. Food intake and oral sucrose in preterm prior to heel prick. Acta Paediatr. 2002;91:555-60.

41. Johnston C, Filion F, Snider L, Majnemer A, Limperopoulos C Walker $C$, et al. Routine sucrose analgesia during the first week of life in neonates younger than 31 weeks' postconceptional age. Pediatrics. 2002;110:523-8.

42. Acharya A, Annamali S, Taub N, Field D. Oral sucrose analgesia for preterm infant venopuncture. Arch Dis Child Fetal Neonatal Ed. $2004 ; 89: 17-8$
43. Boyer $\mathrm{K}$, Johnston $\mathrm{C}$, Walker $\mathrm{CD}$, Filion $\mathrm{F}$, Sherrard A. Does sucrose analgesia promote physiologic stability in preterm neonates? Biol Neonate. 2004;85:26-31

44. Gibbins S, Stevens B, Hodnett E, Pinelli J, Ohlsson A, Darlington $G$. Efficacy and safety of sucrose for procedural pain relief in preterm and term neonates. Nur Res. 2002;51:375-82.

45. Gibbins S, Stevens B. The influence of gestational age on the efficacy and short-term safety of sucrose for procedural pain relief. Adv Neonatal Care. 2003;3:241-9.

\section{Correspondence:}

Maria Beatriz Martins Linhares

Av. João Fiusa, 1725/93

CEP 14024-250 - Ribeirão Preto, SP, Brazil

Tel.: + 55 (16) $625.0309 / 625.0490$

Fax: +55 (16) 625.0309

E-mail: linhares@fmrp.usp.br 